University of Nebraska - Lincoln

DigitalCommons@University of Nebraska - Lincoln

2012

\title{
A quantum-mechanical relaxation model
}

\author{
Ralph Skomski \\ University of Nebraska-Lincoln, rskomski2@unl.edu \\ Arti Kashyap \\ Indian Institute of Technology Mandi, Himachal Pradesh, akashyap@Inmiit.ac.in \\ David J. Sellmyer \\ University of Nebraska-Lincoln, dsellmyer@unl.edu
}

Follow this and additional works at: https://digitalcommons.unl.edu/physicssellmyer

Part of the Physics Commons

Skomski, Ralph; Kashyap, Arti; and Sellmyer, David J., "A quantum-mechanical relaxation model" (2012). David Sellmyer Publications. 237.

https://digitalcommons.unl.edu/physicssellmyer/237

This Article is brought to you for free and open access by the Research Papers in Physics and Astronomy at DigitalCommons@University of Nebraska - Lincoln. It has been accepted for inclusion in David Sellmyer Publications by an authorized administrator of DigitalCommons@University of Nebraska - Lincoln. 


\title{
A quantum-mechanical relaxation model
}

\author{
R. Skomski, ${ }^{1, a)}$ A. Kashyap, ${ }^{2}$ and D. J. Sellmyer ${ }^{1}$ \\ ${ }^{1}$ Department of Physics and Astronomy and Nebraska Center for Materials and Nanoscience, \\ University of Nebraska, Lincoln, Nebraska 68588, USA \\ ${ }^{2}$ School of Basic Sciences, Indian Institute of Technology Mandi, Himachal Pradesh 175001, India
}

(Presented 31 October 2011; received 23 September 2011; accepted 19 December 2011; published online 13 March 2012)

\begin{abstract}
The atomic origin of micromagnetic damping is investigated by developing and solving a quantum-mechanical relaxation model. A projection-operator technique is used to derive an analytical expression for the relaxation time as a function of the heat-bath and interaction parameters. The present findings are consistent with earlier research beyond the Landau-Lifshitz-Gilbert (LLG) equation and show that the underlying relaxation mechanism is very general. Zermelo's recurrence paradox means that there is no true irreversibility in non-interacting nanoparticles, but the corresponding recurrence times are very long and can be ignored in many cases. (C) 2012 American Institute of Physics. [doi:10.1063/1.3679605]
\end{abstract}

\section{INTRODUCTION}

Our understanding of time-dependent magnetization processes is largely based on the Néel-Brown model and on the Landau-Lifshitz-Gilbert equation. The former model is actually a variation of the Arrhenius model, first applied to magnetism around $1930^{1}$ and put on a sound statistical foundation by Kramers in $1940 .^{2}$ Both models are mesoscopic, that is, they employ parameters such as activation energies and damping constants. From a quantum-mechanical point of view, this approach is very crude, and in the 1960s it became clear that there are heat-bath-related atomic contributions such as memory functions. ${ }^{3}$ These corrections are important for the determination of micromagnetic parameters and have a direct impact on magnetization dynamics.

Renewed interest in magnetization dynamics has been sparked by several technological developments. First, magnetic relaxation affects the long-term performance of permanent magnets, by making extrinsic properties such as remanence, coercivity, and energy product time-dependent. ${ }^{4}$ Second, magnetic recording rapidly moves toward very fast magnetization processes, and the understanding of relaxation processes is an important aspect of this development. ${ }^{5}$ Third, in spin electronics it is necessary to consider dissipative spin dynamics, and the exploitation of quantum-mechanical spin effects in quantum computing requires a precise control over decoherence. ${ }^{6}$

A key aspect of relaxation is the involvement of the heat bath, which can be treated numerically, for example by Monte Carlo simulations, or by complicated model calculations. $^{7-9}$ The heat bath and its interactions depend on the physical system, for example on whether the magnetic material is metallic or insulating. ${ }^{9-11}$ Very often, the heat bath degrees of freedom are phononic and the interaction involves spin-orbit coupling, but magnonic processes may also be important. ${ }^{12}$ An example of a recent numerical approach is the use of first-principle scattering theory to calculate the influence of spin-orbit coupling on the damping

\footnotetext{
a) Author to whom correspondence should be addressed. Electronic mail: rskomski@neb.rr.com.
}

parameter $\alpha$ in substitutionally disordered $\mathrm{Ni}_{1-\mathrm{x}} \mathrm{Fe}_{\mathrm{x}} \cdot{ }^{8}$ However, relaxation-time calculations are generally demanding and time consuming, as is the derivation of macroscopic or nanoscale equations of motion from master equations., 9,13

Here we present a physically very transparent and analytically solvable quantum-mechanical relaxation model.

\section{CALCULATION AND RESULTS}

In the modeling, it is important to distinguish between dissipation and dephasing. Both lead to quantum-mechanical decoherence and can be considered as types of relaxation, but the former involves an energy transfer between the magnetic subsystem and the heat bath, which is not necessarily the case for dephasing. A well-known example is the distinction between the respective longitudinal and transversal relaxation times $T_{1}$ and $T_{2}$ in nuclear resonance, where $T_{1} \geq T_{2}$ and often $T_{1} \gg T_{2}{ }^{7}$ because $T_{1}$ requires a transfer of Zeeman energy to the lattice. In the Landau-Lifshitz-Gilbert equation, this distinction is blurred, because the main mechanism is transversal ( $T_{2}$-like) but also contains a dissipative relaxation parallel to the field direction, which is a $T_{1}$ feature. This paper directly focuses on such dissipative relaxation processes, without attempting to further analyze the $T_{1}-T_{2}$ problem.

An alternative view on the relaxation problem is to consider the transition rates in the master equation ${ }^{7}$ that describe the dynamics of the system. Fermi's golden rule means that quantum-mechanical transitions are likely only between degenerate states. In the corresponding microcanonical master equation, there are no transitions that involve Arrheniusor Boltzmann-like terms of the type $\exp \left(-E / k_{\mathrm{B}} T\right)$. Heat-bath master equations, which contain these exponential terms, are obtained by separately considering heat-bath and magnetic degrees of freedom. ${ }^{7}$ The present approach is of the heatbath type, although heat bath and temperature are included only implicitly.

The heat bath and its interaction with the magnetic system are modeled by quantum mechanical matrix elements. The general Hamiltonian is 


$$
\mathcal{H}=\left(\begin{array}{cc}
H_{\mathrm{B}} & \mathrm{V} \\
\mathrm{V}^{+} & H_{\mathrm{M}}
\end{array}\right)
$$

where $H_{\mathrm{B}}$ describes the heat bath, $H_{\mathrm{M}}$ is the Hamiltonian of the magnetic subsystem, and $\mathrm{V}$ is the coupling between heat bath and magnetic degrees of freedom. Figure 1 illustrates the meaning of this equation.

One way of solving Eq. (1) is to use the projectionoperator technique, ${ }^{7}$ by manipulating the Schrödinger equation that belongs to Eq. (1):

$$
\begin{aligned}
& \mathrm{i} \hbar \frac{\partial}{\partial t}\left|\phi>=\mathcal{H}_{\mathrm{B}}\right| \phi>+\mathrm{V} \mid \psi>, \\
& \mathrm{i} \hbar \frac{\partial}{\partial t}\left|\psi>=\mathrm{V}^{+}\right| \phi>+\mathcal{H}_{\mathrm{M}} \mid \psi>.
\end{aligned}
$$

Here the respective heat-bath and magnetic wave functions $|\phi\rangle$ and $|\psi\rangle$ are generally very complicated objects. The idea is to formally solve Eq. (2a) and to insert the resulting $\mid \phi(t)>$ into Eq. (2b). This eliminates the heat-bath degrees of freedom $|\phi\rangle$ but is paid by additional terms in (b). These terms include memory functions, random thermal noise originating from the initial conditions in (a), and - of special interest here-the relaxation time of the magnetic system.

In the present model, the magnetic subsystem is described by a single magnetic state $|\psi\rangle$, where $\langle\psi \mid \psi\rangle$ has the character of a spin density (magnetization) and the relaxation time describes how fast the magnetization is absorbed by the heat. For the heat bath, we assume that the matrix $H_{\mathrm{B}}$ has already been diagonalized. This is meaningful, because the corresponding unitary transformation merely remixes the matrix elements of $\mathrm{V}$ and because our final result will depend on the density of states (DOS) of $H_{\mathrm{B}}$ only. Equation (1) now becomes

$$
\mathcal{H}=\left(\begin{array}{cccccc}
E_{1} & 0 & \cdot & 0 & \cdot & V_{1} \\
0 & E_{2} & \cdot & 0 & \cdot & V_{2} \\
\cdot & \cdot & \cdot & \cdot & \cdot & \cdot \\
0 & 0 & \cdot & E_{\mu} & \cdot & V_{\mu} \\
\cdot & \cdot & \cdot & \cdot & \cdot & \cdot \\
V_{1}^{*} & V_{2}^{*} & \cdot & V_{\mu}^{*} & \cdot & E_{\mathrm{M}}
\end{array}\right) .
$$

In this matrix, the $E_{\mu}$ are the energy eigenvalues of the heat bath.

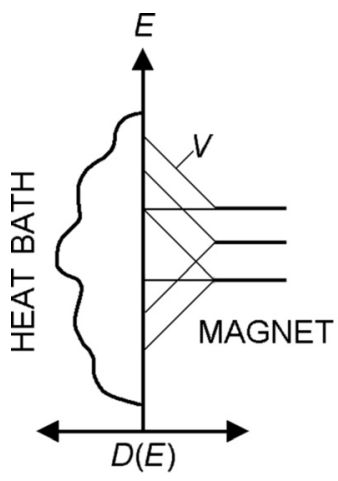

(a)

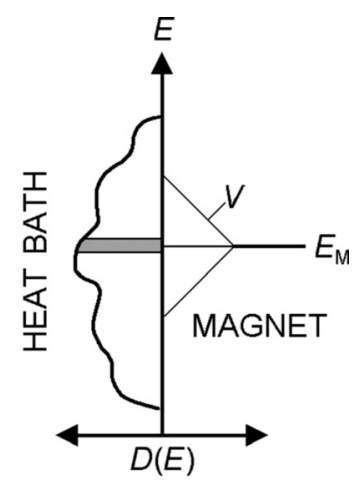

(b)
FIG. 1. Quantum-mechanical origin of relaxation: (a) general case and (b) present model.
The magnetic system is connected by matrix elements $\mathrm{V}_{\mu}$ to each of the heat-bath degree of freedom $(\mu=1 . N, N \rightarrow \infty)$. This involvement of $\mathrm{V}$ is very general and also occurs in real systems, where it describes the effect of the lattice and electronic degrees of freedom on the magnetic subsystem. Combination of Eqs. (2) and (3) yields $N$ equations for the heat-bath wave functions $\left|\phi_{\mu}\right\rangle$. Since $H_{\mathrm{B}}$ is diagonal, these equations are only indirectly coupled, via $V_{\mu}$ and $E_{\mathrm{M}}$, and can be formally solved:

$\phi_{\mu}(t)=\phi_{\mu}(0) \exp \left(-\mathrm{i} \omega_{\mu} \mathrm{t}\right)-\frac{i}{\hbar} \int_{0}^{t} \exp \left(i \omega_{\mu}\left(t^{\prime}-t\right)\right) V_{\mu} \psi\left(t^{\prime}\right) \mathrm{dt}^{\prime}$,

where $\omega_{\mu}=E_{\mu} / \hbar$. Inserting Eq. (4) into the Eq. (2b) and shifting the zero point of the energy scale to $E_{\mathrm{M}}=0$ yields

$$
\begin{aligned}
\mathrm{i} \hbar \frac{\partial}{\partial t} \psi(\mathrm{t})= & \sum_{\mu=1}^{N} V_{\mu}^{*} \phi_{\mu}(0) \exp \left(-\mathrm{i} \omega_{\mu} t\right)-\frac{i}{\hbar} \sum_{\mu=1}^{N} \\
& \times V_{\mu}^{*} \int_{0}^{t} \exp \left(\mathrm{i} \omega_{\mu}\left(t^{\prime}-t\right)\right) V_{\mu} \psi\left(t^{\prime}\right) \mathrm{d} t^{\prime} .
\end{aligned}
$$

The first term on the right-hand side of this equation is the thermal noise caused by the randomness of the initial condition $\phi_{\mu}(0)$ of the heat-bath, which is assumed to be in thermal equilibrium at some temperature $T$.

The second term yields the damping constant or relaxation time. Writing the summation as an integral, $\Sigma_{\mu} \mathrm{V}_{\mu} * \mathrm{~V}_{\mu}$. $\rightarrow \int \mathrm{D}(\omega) \mathrm{V}^{2}(\omega)$. $\mathrm{d} \omega$, we obtain

$$
\begin{aligned}
\mathrm{i} \hbar \frac{\partial}{\partial t} \psi(t)= & \mathrm{i} \hbar f_{\mu}(\mathrm{t})-\frac{i}{\hbar} \int_{-\infty}^{\infty} D(\omega) V^{2}(\omega) \\
& \times \int_{0}^{t} \exp \left(\mathrm{i} \omega\left(t^{\prime}-t\right)\right) \psi\left(t^{\prime}\right) \mathrm{d} t^{\prime} \mathrm{d} \omega,
\end{aligned}
$$

where $f_{\mu}(\mathrm{t})$ describes the thermal noise. This integral is nearly exclusively determined heat-bath degrees of freedom with $\omega \approx 0$, or $E_{\mu} \approx E_{\mathrm{M}}$. Physically, Fermi's golden rule means that transitions between quantum states are most pronounced if they have the same energy. In the present case, the total energy is conserved, but there is an energy exchange between heat bath and magnetic subsystem.

The leading role of the contributions from $\omega \approx 0$ allows us to replace the integral over $\omega$ by a delta function and yields

$$
\frac{\partial}{\partial t} \psi(t)+\Gamma \psi(t)=f_{\mu}(\mathrm{t})
$$

where the relaxation rate or inverse relaxation time $\Gamma=1 / \tau$ is given by

$$
\Gamma=\frac{\pi}{\hbar} D\left(E_{\mathrm{M}}\right) V^{2}\left(E_{\mathrm{M}}\right) .
$$

This shows that the relaxation rate is proportional to the density of states of the heat bath and increases quadratically with the coupling strength between magnet and heat bath. Note that the replacement of the wave function $|\psi\rangle$ by quantum-mechanical averages, $\langle\psi|\mathrm{A}| \psi\rangle$, does not change the involvement of the relaxation time. ${ }^{13}$

Note that Eq. (8) contains the density of states (DOS) of the heat bath but not the DOS of the magnetic subsystem. This asymmetry is caused by the thermodynamic limit of the heat bath and also occurs in more complicated model systems. ${ }^{7}$ Physically, it reflects the transition from the microcanonical 


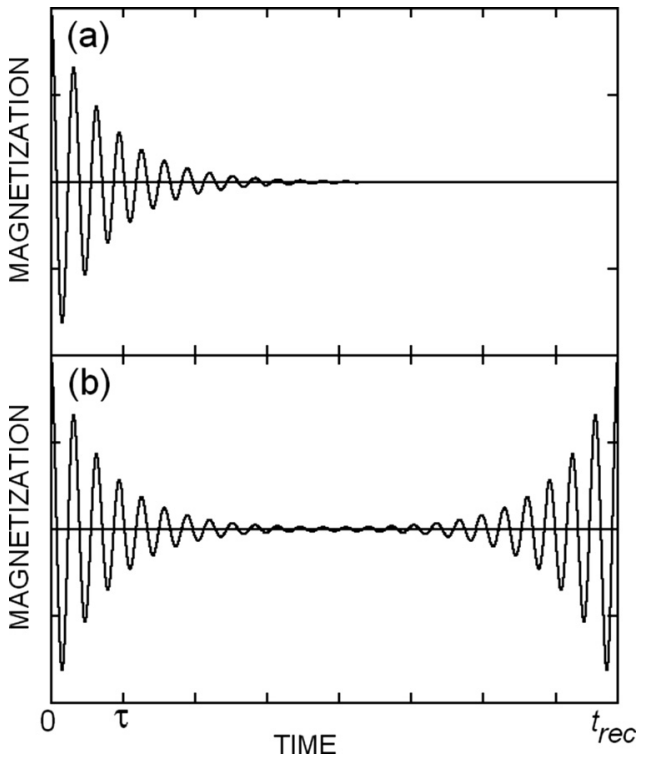

FIG. 2. Zermélo's recurrence paradox in a magnetic nanoparticle: (a) irreversible relaxation and (b) recurrence.

Pauli master equation, which directly obeys Fermi's golden rule, to a heat-bath master equation.

The findings of this section are consistent with earlier research on the topic, ${ }^{7}$ and there is even a classical mechanical model with a very similar behavior. ${ }^{14}$ This shows that the simple picture of Fig. 2(b) captures the essential physics of irreversibility and relaxation.

\section{DISCUSSION AND CONCLUSIONS}

The simplicity and transparence of the present model is paid by a relatively poor description of both heat bath and spin system. The former is not very critical, and it is known that it does not even matter very much whether the heat bath is treated classically or quantum-mechanically. ${ }^{7}$ In the present model, the temperature is contained in the thermal noise $f_{\mu}(\mathrm{t})$, Eq. (6). The noise term is physically similar to the randomforce term in Langevin-type equations and originates from the thermally randomized initial condition $\phi_{\mu}(0)$ of the heat bath.

Our model has no magnetization vector and no spin precession, but LLG damping can be discussed in terms of relaxation times very similar to the present one. ${ }^{13}$ Basically, the inverse relaxation time $\Gamma$ is the product of the LLG damping constant and precession frequency. To include spin precession in Eq. (2), it would be necessary to replace the scalar $E_{M}$ by a Pauli-type $2 \times 2$ matrix. The algebra of the Pauli matrices involve cross-products that translate into LLG precession, but the interaction of these precession modes with the heat bath is a rather complicated problem even for single spins. ${ }^{7}$

As any other finite systems, isolated magnetic nanoparticles are characterized by discrete energy levels, which makes it impossible to perform the transition to a continuous density of states, Eq. (6), and to derive a relaxation time. This is different from a nanoparticle or spin in coupled to the environment, where the magnetic degrees of freedom are dis- crete but the heat bath described by a continuous DOS, as in Eq. (8). Let us assume, for simplicity, that the dynamics of the $i$ th particle is oscillatory, $m_{\mathrm{i}}=m_{\mathrm{o}} \cos \left(\omega_{\mathrm{i}} t\right)$, and that the nanoparticle ensemble can be described by a distribution $P(\omega)$. The ensemble-averaged magnetization is then

$$
<m(t)>=m_{\mathrm{o}} \int P(\omega) \cos (\omega t) \mathrm{d} \omega .
$$

For a continuous distribution $\mathrm{P}(\mathrm{w}),<m(t)>$ is damped with a relaxation time of the order of $1 / \Delta \omega$, where $\Delta \omega$ is the width of the frequency distribution. Replacing the continuous function $P(\omega)$ by a sum of delta functions, as appropriate for nanoparticles, yields a recurrence time $t \sim 1 / \delta \omega$, where $\hbar \delta \omega$ is some characteristic energy-level spacing (Fig. 2). In a more general context, this is known as Zermélo's recurrence paradox. ${ }^{15}$ However, the recurrence time is usually very long, even in small nanoparticles, because the level splitting $\hbar \delta \omega$ rapidly decreases with increasing particle size.

In conclusion, we have developed and analytically solved a simple quantum-mechanical relaxation model. As more complicated approaches, it reproduces the main features of the relaxation, namely the involvement of the heat bath's density of states and interactions with the magnetic subsystem. Our focus has been on the relaxation time, but the model also yields a memory effect that goes beyond the LLG equations. Due to Zermelo's paradox, the present approach cannot be applied to isolated nanoparticles, but the corresponding recurrence times are very long and can often be ignored, even for small nanoparticles.

\section{ACKNOWLEDGMENTS}

Thanks are due to P. Lougovski for discussing decoherence effects in quantum systems. This research is supported by NSF-MRSEC (RS), DOE (DJS), BREM (RS), DST (AK), and NCMN.

${ }^{1}$ R. Becker and W. Döring, Ferromagnetismus (Springer, Berlin, 1939).

${ }^{2}$ H. A. Kramers, Physica 7, 284 (1940).

${ }^{3}$ R. Zwanzig, Phys. Rev. 124, 983 (1961).

${ }^{4}$ R. Skomski and J. M. D. Coey, Permanent Magnetism (Institute of Physics, Bristol, UK, 1999).

${ }^{5}$ D. Weller and T. McDaniel, in Advanced Magnetic Nanostructures, edited by D. J. Sellmyer and R. Skomski, (Springer, Berlin, 2006), Ch. 11, p. 295.

${ }^{6}$ M. A. Nielsen and I. L. Chuang, Quantum Computation and Quantum Information (University Press, Cambridge, UK, 2000).

${ }^{7}$ R. Zwanzig, Nonequilibrium Statistical Mechanics (University Press, Oxford, UK, 2001).

${ }^{8}$ A. A. Starikov, P. J. Kelly, A. Brataas, Y. Tserkovnyak, and G. E. W. Bauer, Phys. Rev. Lett. 105, 236601 (2010).

${ }^{9}$ M. Fähnle and C. Illg, J. Phys.: Condens. Matter 23, 493201 (2011).

${ }^{10}$ V. Kambersky and C. E. Patton, Phys. Rev. B 11, 2668 (1975).

${ }^{11}$ B. Heinrich, R. Urban, and G. Woltersdorf, IEEE Trans. Magn. 38(5), 2496 (2002)

${ }^{12}$ H. Suhl, IEEE Trans. Magn. 34(4), 1834 (1998).

${ }^{13}$ R. Skomski, J. Zhou, and D. J. Sellmyer, J. Appl. Phys. 97, 10 A702 (2005).

${ }^{14}$ R. Skomski, Simple Models of Magnetism (University Press, Oxford, UK, 2008).

${ }^{15} \mathrm{~W}$. Brenig, Statistical Theory of Heat: Nonequilibrium Phenomena (Springer, Berlin, 1989). 